04

\title{
Проникновение пробного поля в греющуюся плазму
}

\author{
(C) К.Н. Овчинников, С.А. Урюпин \\ Физический институт им. П.Н. Лебедева РАН, \\ 119991 Москва, Россия \\ ฯ e-mail: uryupin@sci.lebedev.ru
}

(Поступило в Редакцию 6 июля 2016 г. В окончательной редакции 12 октября 2016 г.)

Найдены явные зависимости эффективной глубины проникновения поля пробного импульса в токонесущую плазму с изменяющимися во времени температурами частиц. Показано, что проникновение поля в полностью ионизованную плазму происходит в режиме субдиффузии, а в слабоионизованную — в режиме сверхдиффузии.

DOI: 10.21883/JTF.2017.06.44505.1971

\section{Введение}

Импульсы электромагнитного поля используются для диагностики токонесущей плазмы и ее дополнительного нагрева. Для выбора оптимальных условий диагностики и нагрева нужно знать закономерности проникновения импульса в ограниченную плазму. Прежде всего эти закономерности зависят от того, какой механизм рассеяния электронов доминирует во время воздействия импульса. В плазме с большой плотностью тока, когда дрейфовая скорость электронов превосходит фазовую скорость ионно-звуковых волн, основной причиной рассеяния электронов являются флуктуации плотности заряда в поле ионно-звуковых волн [1-3]. Особенности проникновения монохроматического поля в турбулентную плазму исследованы в работах [4-7]. Изучению проникновения квазистационарного сильного и слабого полей в плазму с развитой ионно-звуковой турбулентностью посвящены работы $[8,9]$. При этом в [9] показано, что закономерности проникновения слабого импульса в значительной мере зависят от соотношения его длительности и характерного времени изменения температур частиц, а также от направления слабого поля относительного греющего плазму поля. Следует отметить, что в экспериментах встречаются и такие условия, когда дрейфовая скорость электронов сравнима со скоростью ионного звука (см., например, [10]) или меньше ее. В последнем случае доминирующим механизмом рассеяния электронов являются их столкновения с ионами. При еще меньших плотностях тока газ лишь частично ионизован и возможны условия, в которых доминируют столкновения электронов с нейтральными частицами. Несмотря на то что разряды с небольшой плотностью тока используются многие годы, задача об особенностях воздействия слабых пробных импульсов на такие разряды, по-видимому, не ставилась. Вместе с тем такая задача представляет определенный интерес не только сама по себе, но и в связи с необходимостью сопоставления особенностей взаимодействия слабых импульсов с ламинарной и турбулентной плазмами.
В настоящем сообщении изучено проникновение слабого импульса в токонесущую плазму с изменяющимися во времени температурами частиц. Для полностью ионизованной и слабоионизованной плазм установлены явные зависимости эффективной глубины проникновения поля от времени, параметров плазмы и импульса. Показано, что в случае полностью ионизованной плазмы проникновение поля длинного импульса происходит в режиме субдиффузии, когда эффективная глубина проникновения зависит от времени как $t^{1 / 5}$. В слабоионизованной плазме, в которой доминирует упругое рассеяние электронов на нейтральных частицах, глубина проникновения поля пропорциональна $t$, что позволяет говорить о сверхдиффузии поля. Отметим, что выявленные закономерности проникновения отличаются от закона, установленного ранее для турбулентной плазмы, в которой глубина проникновения возрастает пропорционально $t^{1 / 4}[9]$. Иными являются и явные зависимости глубины проникновения от параметров плазмы, что позволяет выбирать оптимальные условия не только диагностики токонесущей плазмы, но и ее дополнительного нагрева.

\section{1. Эволюция температур частиц}

Пусть плазма занимает полупространство $x>0$. Примем, что в плазме имеется однородное электрическое поле вида $\mathbf{E}=(0,0, E)$, которое создает зависящий от времени электрический ток, плотность которого $\mathbf{j}=(0,0, j)$. Однородный ток связан с полем соотношением

$$
4 \pi j+\frac{\partial}{\partial t} E=\text { const. }
$$

Как правило, в плазме ток смещения мал по сравнению с током проводимости, и им можно пренебречь. При этом вместо уравнения (1) приближенно имеем $j \sim$ const. Явный вид зависимости тока от электрического поля определяется материальными уравнениями. Ниже рассмотрены полностью ионизованная и слабоионизованная плазмы. Закономерности диссипативных процессов в плазме с током зависят от величины токовой скорости 
электронов $u$, которая связана с плотностью тока соотношением

$$
u=j / e n_{e},
$$

где $e$ и $n_{e}$ - заряд и плотность электронов. В дальнейшем будем рассматривать условия, в которых токовая скорость электронов меньше скорости ионного звука $u<v_{s}$.

\section{1. Полностью ионизованная плазма}

В однородной плазме эволюция температур электронов $T_{e}$ и ионов $T_{i}$ описывается уравнениями

$$
\begin{gathered}
\frac{d T_{e}}{d t}=\frac{2 \mathbf{j} \mathbf{E}}{3 n_{e} æ}-v_{\varepsilon}\left(T_{e}-T_{i}\right), \\
\frac{d T_{i}}{d t}=v_{\varepsilon} Z\left(T_{e}-T_{i}\right) .
\end{gathered}
$$

В этих уравнениях $\mathbf{j}=\sigma \mathbf{E}-$ плотность тока, æ постоянная Больцмана,

$$
\sigma=32 e^{2} n_{e} /\left(3 \pi m_{e} v_{e i}\right)
$$

- проводимость плазмы, а эффективные частоты столкновений имеют вид

$$
v_{\varepsilon}=\frac{2 m_{e}}{m_{i}} v_{e i}, \quad v_{e i}=\frac{4 \sqrt{2 \pi}}{3} \frac{e^{2} e_{i}^{2} n_{i} \Lambda}{m_{e}^{2} v_{T e}^{3}},
$$

где $v_{e i}$ - частота электрон-ионных столкновений, $v_{T e}=\sqrt{æ T_{e} / m_{e}}-$ тепловая скорость электронов, $\Lambda$ кулоновский логарифм, $Z=\left|e_{i} / e\right|$, а $m_{e}$ и $m_{i}-$ массы электрона и иона, $e_{i}$ и $n_{i}$ - заряд и плотность ионов. Первое слагаемое в правой части уравнения (3) описывает джоулев нагрев, второе - передачу энергии от электронов к ионам. Примем, что в начальный момент времени температуры электронов и ионов одинаковы $T_{i}=T_{e}=T_{0}$.

Тогда, когда $j=$ const, система уравнений $(3),(4)$ для температур принимает вид

$$
\begin{gathered}
\frac{d T_{e}}{d \tau_{i}}=\left(T_{0} / T_{e}\right)^{3 / 2}\left[a T_{0}-\left(T_{e}-T_{i}\right)\right], \\
\frac{d T_{i}}{d \tau_{i}}=Z\left(T_{0} / T_{e}\right)^{3 / 2}\left(T_{e}-T_{i}\right) .
\end{gathered}
$$

При написании уравнений (7) и (8) использованы обозначения

$$
\tau_{i}=v_{\varepsilon}(0) t,
$$

где $v_{\varepsilon}(0)$ - частота релаксации температур в начальный момент времени и

$$
a=\frac{\pi}{32} \frac{m_{i}}{m_{e}}\left[\frac{u}{v_{T_{0}}}\right]^{2}=\frac{\pi Z}{32}\left[\frac{u}{v_{s}(0)}\right]^{2} \ll 1,
$$

где $v_{T_{0}}=\sqrt{\mathfrak{x} T_{0} / m_{e}}, v_{s}(0)=\sqrt{Z æ T_{0} / m_{i}}$. Вводя переменную $\Omega$, согласно соотношению $d \Omega / d \tau_{i}=\left(T_{0} / T_{e}\right)^{3 / 2}$, из системы уравнений (7) и (8) находим

$$
Z T_{e}+T_{i}=a Z T_{0} \Omega+(1+Z) T_{0},
$$

$$
T_{e}-T_{i}=\frac{a T_{0}}{1+Z}\{1-\exp [-(1+Z) \Omega]\}
$$

По определению, функция $\Omega$ монотонно возрастает со временем. В частности, при $(1+Z) \Omega \ll 1$ из $(11),(12)$ имеем $T_{e} \sim T_{0}(1+a \Omega)$ и $T_{i} \sim T_{0}$. Если же $(1+Z) \Omega \gg 1$, Tо

$$
\begin{gathered}
T_{e}-T_{i} \approx \frac{a T_{0}}{1+Z}, \\
T_{e} \approx T_{0}\left(1+\frac{Z}{1+Z} a \Omega\right) .
\end{gathered}
$$

Из соотношений (10)-(14) видно, что температура электронов остается близкой к исходной $T_{0}$, если $a \Omega \ll 1$. Это означает, что при $(1+Z) \Omega \ll 1$ отличием $\Omega$ и $\tau_{i}$ можно пренебречь. Далее, используя соотношение (13) и факт близости температуры $T_{e}$ к $T_{0}$ в момент, когда $(1+Z) \tau_{i} \sim 1$, из уравнения (7) приближенно находим

$$
T_{e} \approx T_{0}\left[1+\frac{5 Z a \tau_{i}}{2(1+Z)}\right]^{2 / 5} .
$$

При $(1+Z) \tau_{i} \gtrsim 1$ температура ионов увеличивается по такому же закону, оставаясь меньше $T_{e}$ на малую величину $a T_{0} /(1+Z)($ см. (13)). Существенное увеличение температур начинается с момента

$$
\tau_{i} \approx \frac{2(1+Z)}{5 Z a} \gg \frac{1}{1+Z}
$$

или в явном виде при

$$
\begin{aligned}
t \gtrsim t_{T} & \equiv \frac{32}{5 \pi} \frac{1+Z}{Z}\left[\frac{v_{T_{0}}}{u}\right]^{2} \frac{1}{v_{e i}(0)} \\
& =\frac{64}{5 \pi} \frac{1+Z}{Z^{2}}\left[\frac{v_{s}(0)}{u}\right]^{2} \frac{1}{v_{\varepsilon}(0)} \gg \frac{1}{v_{\varepsilon}(0)},
\end{aligned}
$$

где $t_{T}-$ характерное время нагрева электронов и ионов, $v_{e i}(0)$ - частота столкновений электронов с ионами при $T_{e}=T_{0}$.

\section{2. Слабоионизованная плазма}

В слабоионизованной плазме рассеяние электронов в основном происходит на нейтральных частицах. Для частоты упругих столкновений электронов с тяжелыми нейтральными частицами используем простейшее выражение $v_{e n}(v)=N \Sigma v$, где $N-$ плотность нейтральных частиц, а $\Sigma$ - не зависящее от скорости электрона сечение рассеяния. Тогда для проводимости имеем

$$
\sigma=\frac{2}{3} \sqrt{\frac{2}{\pi}} \frac{e^{2} n_{e}}{m_{e} v_{e n}}
$$

где $v_{e n}=v_{e n}\left(v_{T_{e}}\right)$. Используя приведенное в [11] (см. формулы (2.5) на стр. 215, (2.11) на стр. 218, (2.13) на стр. 219) кинетическое уравнение для изотропной части 
функции распределения электронов и считая распределение электронов близким к максвелловскому, запишем уравнение для температуры электронов

$$
\frac{d T_{e}}{d t}=\frac{2 \mathbf{j} \mathbf{E}}{3 n_{e} æ}-v_{\varepsilon n}\left(T_{e}-T\right),
$$

где использовано обозначение

$$
v_{\varepsilon n}=\frac{16}{3 \sqrt{2 \pi}} \frac{2 m_{e}}{M} v_{e n} .
$$

Здесь $M$ - масса нейтральной частицы. При этом для $T$ - температуры нейтральных частиц имеем

$$
\frac{d T}{d t}=v_{\varepsilon n}\left(T_{e}-T\right)
$$

В условиях, когда $j=$ const, представим уравнения (19), (21) в виде

$$
\begin{gathered}
\frac{d T_{e}}{d \tau_{n}}=\sqrt{T_{e} / T_{0}}\left[a_{n} T_{0}-\left(T_{e}-T\right)\right] \\
\frac{d T}{d \tau_{n}}=\sqrt{T_{e} / T_{0}}\left(T_{e}-T\right),
\end{gathered}
$$

где $\tau_{n}-$ безразмерное время,

$$
\tau_{n}=v_{\varepsilon n}(0) t,
$$

$v_{\varepsilon n}(0)$ - частота релаксации температуры $v_{\varepsilon n}$ в начальный момент времени, когда $T_{e}=T=T_{0}$, а параметр $a_{n}$ имеет вид

$$
a_{n}=\frac{3 \pi}{16} \frac{M}{2 m_{e}}\left[\frac{u}{v_{T_{0}}}\right]^{2} \ll 1 .
$$

Вводя переменную $\Omega_{n}$, согласно соотношению $d \Omega_{n} / d \tau_{n}=\sqrt{T_{e} / T_{0}}$, из системы уравнений (22) и (23) находим

$$
\begin{gathered}
T_{e}+T=2 T_{0}\left(1+a_{n} \Omega_{n} / 2\right), \\
T_{e}-T=0.5 a_{n} T_{0}\left[1-\exp \left(-2 \Omega_{n}\right)\right] .
\end{gathered}
$$

Тогда, когда $\Omega_{n} \ll 0.5$, из (26) и (27) получаем $T_{e} \sim T_{0}\left(1+\Omega_{n} a_{n}\right), T \sim T_{0}$. Из этих соотношений и определения $\Omega_{n}$ видим, что при $\Omega_{n} \ll 0.5$ имеет место связь $\Omega_{n} \sim \tau_{n}$. Если $\Omega_{n} \gg 0.5$ или $\tau_{n} \gg 0.5$, из (27) приближенно имеем

$$
T_{e}-T \approx 0.5 a_{n} T_{0}
$$

Используя соотношение $(28)$ и условие $T_{e}\left(\tau_{n} \sim 0.5\right) \sim$ $\sim T_{0}\left(1+0.5 a_{n}\right) \sim T_{0}$ из уравнения (22) для температуры электронов, находим

$$
\begin{aligned}
T_{e} & =T_{0}\left[\sqrt{1+0.5 a_{n}}+a_{n}\left(\tau_{n}-0.5\right) / 4\right]^{2} \\
& \approx T_{0}\left(1+a_{n} \tau_{n} / 4\right)^{2}
\end{aligned}
$$

При этом температура электронов незначительно превышает температуру нейтральных частиц (см. (28)).
Характерное время нагрева частиц в слабоионизованной плазме дается соотношением $\tau_{n} \sim 4 / a_{n}$ или

$$
t_{T_{n}}=4 \sqrt{\frac{2}{\pi}}\left[\frac{v_{T_{0}}}{u}\right]^{2} \frac{1}{v_{e n}(0)}
$$

где $v_{e n}(0)=v_{e n}\left(v_{T_{0}}\right)$.

Полученные выше закономерности эволюции температуры электронов (см. (15) и (29)) позволяют описать влияние нагрева электронов на проникновение пробного импульса.

\section{2. Воздействие пробного импульса}

Примем, что на плазму перпендикулярно ее границе падает электромагнитный импульс вида

$$
\begin{aligned}
E_{y}^{(i)}(x, t)= & E_{p y}[\eta(t-x / c-\Delta) \\
& \left.-\eta\left(t-x / c-\Delta-t_{p}\right)\right], \quad x<0,
\end{aligned}
$$

где $\eta(\xi)=0$ при $\xi<0$ и $\eta(\xi)=1$ при $\xi>0, c-$ скорость света, $\Delta-$ время задержки, которое введено для разнесения во времени начала эволюции основного состояния плазмы и начала воздействия электромагнитного импульса, $t_{p}$ - длительность импульса. Электромагнитный импульс частично отражается от плазмы. Внутри плазмы импульс создает возмущение напряженности электрического поля $\delta E_{y}$, которое перпендикулярно основному полю $E$. В линейном приближении по $\delta E_{y}$ возмущения поля $\delta E_{y}$ не приводят к возмущению температур частиц $\delta T_{e}=\delta T_{i}=\delta T=0$. Для определения $\delta E_{y}$ используем уравнение

$$
\frac{\partial^{2}}{\partial x^{2}} \delta E_{y}=\frac{4 \pi}{c^{2}} \frac{\partial}{\partial t}\left(\sigma \delta E_{y}\right)
$$

в которое входит невозмущенная проводимость плазмы $\sigma=j / E$. Локальная связь плотности тока с напряженностью поля имеет место в условиях нормального скин-эффекта. В связи с этим основанное на уравнении (32) описание воздействия пробного импульса на плазму оправдано, если его длительность много больше времени свободного пробега электронов, а длина свободного пробега электронов меньше характерного пространственного масштаба изменения поля. При написании уравнения (32) отброшен ток смещения, который пренебрежимо мал в условиях, когда $4 \pi \sigma t_{E} \gg 1$, где $t_{E}$ - характерное время изменения поля. Уравнение (32) следует дополнить начальным и граничными условиями. В момент $t=0$ считаем, что $\delta E_{y}(x, t=0)=0, x>0$. Достаточно далеко от границы плазмы имеем условие

$$
\delta E_{y}(x \rightarrow \infty, t)=0 .
$$

В свою очередь, из условий непрерывности тангенциальных компонент поля на границе плазмы имеем

$$
\left.\left(\frac{\partial}{\partial t}-c \frac{\partial}{\partial x}\right) \delta E_{y}(x, t)\right|_{x=0}=2 \frac{\partial}{\partial t} E_{y}^{(i)}(x=0, t) .
$$


Тогда, когда мал ток смещения, принимая во внимание явный вид импульса (31) из (34), имеем

$$
\left.\frac{\partial}{\partial x} \delta E_{y}(x, t)\right|_{x=0}=-\frac{2}{c} E_{p y}\left\{\delta(t-\Delta)-\delta\left(t-\Delta-t_{p}\right)\right\} .
$$

С целью решения уравнения (32) перейдем к новой функции $w$ и новой независимой переменной $\tau$, которые определяются соотношениями:

$$
\begin{gathered}
w=w(x, \tau)=\sigma[t(\tau)] \delta E_{y}[x, t(\tau)], \\
\frac{d \tau}{d t}=\frac{1}{4 \pi \sigma(t)}, \quad \tau(t=0)=0 .
\end{gathered}
$$

При этом для определения функции $w(x, \tau)$ имеем уравнение

$$
\frac{\partial}{\partial \tau} w(x, \tau)=c^{2} \frac{\partial^{2}}{\partial x^{2}} w(x, \tau),
$$

граничные условия

$$
\begin{gathered}
w(x \rightarrow \infty, \tau)=0 \\
\left.\frac{\partial}{\partial x} w(x, \tau)\right|_{x=0}=-\frac{2}{c} \sigma[t(\tau)] E_{p y}\{\delta[t(\tau)-\Delta] \\
\left.-\delta\left[t(\tau)-\Delta-t_{p}\right]\right\} \equiv \Psi(\tau)
\end{gathered}
$$

и начальное условие $w(x>0, \tau=0)=0$. Отвечающее таким условиям решение уравнения (38) имеет вид

$$
w(x, \tau)=-\frac{c}{\sqrt{\pi}} \int_{0}^{\tau} \frac{d \tau^{\prime} \Psi\left(\tau^{\prime}\right)}{\sqrt{\tau-\tau^{\prime}}} \exp \left[-\frac{x^{2}}{4 c^{2}\left(\tau-\tau^{\prime}\right)}\right],
$$

что позволяет записать следующее выражение для возмущения поля в плазме:

$$
\begin{aligned}
& \delta E_{y}(x, t)=\frac{E_{p y}}{2 \pi \sqrt{\pi} \sigma(t)} \\
& \times\left\{\frac{\eta(t-\Delta)}{\sqrt{\tau(t)-\tau(\Delta)}} \exp \left[-\frac{x^{2}}{4 c^{2}(\tau(t)-\tau(\Delta))}\right]\right. \\
& \left.-\frac{\eta\left(t-\Delta-t_{p}\right)}{\sqrt{\tau(t)-\tau\left(\Delta+t_{p}\right)}} \exp \left[-\frac{x^{2}}{4 c^{2}\left(\tau(t)-\tau\left(\Delta+t_{p}\right)\right)}\right]\right\} .
\end{aligned}
$$

Согласно (42), проникновение поля ступенчатого импульса в глубь плазмы можно представить как распространение двух фронтов - „включения“ и „выключения“". Фронт „включения“ описывает первое слагаемое в (42), а фронт „выключения“ - второе. Второй фронт смещен по отношению к первому на длительность импульса $t_{p}$. В тех точках, куда пришел второй фронт, происходит частичная компенсация поля. Характерная глубина проникновения поля в плазму определяется первым слагаемым в (42) и дается соотношением

$$
x \propto 2 c \sqrt{\tau(t)-\tau(\Delta)} .
$$

Если проводимость плазмы не изменяется со временем, то, согласно (37), $\tau \propto t$ и проникновение поля в глубь плазмы происходит в соответствии со скейлингом (43), отвечающим обычной диффузии $x \propto \sqrt{t}$. Формулы (37) и (43) позволяют качественно описать проникновение поля в нестационарную среду, когда ее проводимость изменяется со временем из-за нагрева. Если нагрев среды приводит к росту проводимости, то, согласно (37), с течением времени скорость роста $\tau$ уменьшается, т.е. $\tau$ растет медленнее, чем $t$. При этом из соотношения (43) следует, что глубина проникновения растет медленнее, чем $\sqrt{t}$. В этом случае можно говорить о субдиффузии поля. Напротив, если нагрев приводит к уменьшению проводимости, то реализуется рост $\tau$ более быстрый, чем $t$. В этих условиях имеет место сверхдиффузия, т. е. более быстрое проникновение поля по сравнению с обычной диффузией.

\section{3. Влияние нагрева частиц на проникновение поля}

Для получения количественных закономерностей проникновения поля пробного импульса в греющуюся плазму достаточно найти явную связь $\tau(t)$ со временем $t$. Эта связь зависит от вида проводимости $\sigma(t)$, которая отличается в полностью ионизованной и слабоионизованной плазмах.

\section{1. Полностью ионизованная плазма}

В случае полностью ионизованной плазмы имеем $\sigma(t) \propto\left[T_{e}(t) / T_{0}\right]^{3 / 2}$. При этом зависимость температуры электронов от времени дается приближенным соотношением (15). Используя соотношение (15), из уравнения (37) находим, что определяющая глубину проникновения поля (43) разность $\tau(t)-\tau(\Delta)$ имеет вид

$$
\begin{aligned}
\tau(t) & -\tau(\Delta)=\frac{15 \pi}{64} \frac{v_{e i}(0) t_{T}}{\omega_{L e}^{2}} \\
& \times\left[\left(1+t / t_{T}\right)^{2 / 5}-\left(1+\Delta / t_{T}\right)^{2 / 5}\right], \quad t>\Delta .
\end{aligned}
$$

На временах, удовлетворяющих неравенству (17) и при $\Delta>t_{T}$, единицами в круглых скобках формулы (44) можно пренебречь. При этом в соответствии с соотношением (43) в условиях нагрева частиц плазмы для характерной глубины проникновения поля в плазму находим

$$
x \propto \frac{\sqrt{15 \pi}}{4} \frac{c}{\omega_{L e}} \sqrt{v_{e i}(0) t}\left(\frac{t_{T}}{t}\right)^{3 / 10} \sqrt{1-(\Delta / t)^{2 / 5}} .
$$

Из (45) видно, что нагрев плазмы приводит к относительному замедлению проникновения пробного поля в плазму. 


\section{2. Слабоионизованная плазма}

В слабоионизованной плазме температура электронов описывается выражением (29), которое позволяет видеть, как изменяется проводимость $\sigma(t) \propto \sqrt{T_{0} / T_{e}(t)}$. Используя результат (29) из уравнения (37), для разности $\tau(t)-\tau(\Delta)$ получаем

$$
\tau(t)-\tau(\Delta)=\frac{3 \sqrt{\pi}}{2 \sqrt{2}} \frac{\nu_{e n}(0)(t-\Delta)}{\omega_{L e}^{2}}\left[1+\frac{t+\Delta}{2 t_{T n}}\right], t>\Delta .
$$

На стадии нагрева, когда $t+\Delta \gg 2 t_{T_{n}}$, из (46) и (43) получаем зависимость от времени глубины проникновения поля в виде

$$
x \propto(3 \sqrt{\pi / 2})^{1 / 2} \frac{c}{\omega_{L e}} \sqrt{v_{e n}(0) t}\left(\frac{t}{t_{T n}}\right)^{1 / 2} \sqrt{1-(\Delta / t)^{2}} .
$$

В этом случае нагрев плазмы приводит к более быстрому проникновению поля, чем в случае обычной диффузии.

\section{Заключение}

Во время воздействия короткого импульса, когда плазму можно считать стационарной средой, проникновение поля происходит по обычному диффузионному закону $\sqrt{t}$. Учет нагрева плазмы за время действия длинного импульса приводит к изменению закономерностей проникновения поля пробного импульса. В случае полностью ионизованной плазмы, когда доминируют парные электрон-ионные столкновения, проникновение поля происходит в режиме субдиффузии. Если же преобладают упругие столкновения электронов с нейтральными частицами, что имеет место в слабоионизованной плазме, то реализуется сверхдиффузия поля.

В [10] приведены экспериментальные данные, полученные на установке ТУМАН-3, среди которых есть такие, когда токовая скорость электронов сравнима или меньше скорости ионного звука. В этой же работе сопоставлены экспериментальные данные для токамаков с существенно отличающимися размерами и концентрациями плазмы. При этом отмечается, что часть разрядов на проанализированных установках находится в „дозвуковом“ режиме. Дозвуковой режим создавался увеличением плотности числа частиц путем инжекции пеллет (Alcator, ASDEX) либо газонапуска (ASDEX, TFTR). В [10] приведена связь тока и концентрации плазмы на установке DITE для разрядов в дейтерии и водороде. Отмечено, что в обоих случаях получение максимальной концентрации соответствовало фазе с уменьшающимся током и в этой же фазе происходил переход в ,дозвуковой“ режим. Изложенная выше теория может быть использована для анализа проникновения электромагнитного импульса в ,дозвуковые“ разряды на указанных установках, если длительность импульса превышает характерное время нагрева частиц плазмы.
Для оценок используем следующий набор параметров: $n_{e} \sim 6 \cdot 10^{13} \mathrm{~cm}^{-3}, T_{e} \sim 50 \mathrm{eV}, I \sim 100 \mathrm{kA}$ и радиус шнура плазмы $r \sim 15 \mathrm{~cm}$. При таких значениях параметров токовая скорость электронов меньше скорости ионного звука. При этом длина свободного пробега электронов сравнима с $x_{T}$, и для оценок можно использовать соотношения, полученные в предположении о нормальном скин-эффекте. В этих условиях для характерного времени нагрева частиц плазмы и характерного пространственного масштаба $x_{T}=\left(15 \pi v_{e i}(0) t_{T} / 16\right)^{1 / 2} c / \omega_{L_{e}}$ получаем оценки $t_{T} \sim 0.3 \mathrm{~ms}, x_{T} \sim 5 \mathrm{~cm}$. Т. е. представленная теория необходима, если длительность воздействующего импульса превышает $0.3 \mathrm{~ms}$.

В качестве физического объекта для иллюстрации проникновения электромагнитного импульса в слабоионизованную плазму рассмотрим разряд молнии. В разряде молнии величина тока, плотность и температура электронов могут меняться в очень широких пределах. Для оценки примем $n_{e} \sim 10^{16} \mathrm{~cm}^{-3}, T_{e} \sim 1 \mathrm{eV}$. Радиус канала молнии оценим как $r \sim 10 \mathrm{~cm}$ ([12], стр. 134-135), а величину тока как $I \sim 100 \mathrm{kA}$ ([12], стр. 122). Для оценки также примем $\Sigma \sim 10^{-15} \mathrm{~cm}^{2}$ и $N \sim 3 \cdot 10^{19} \mathrm{~cm}^{-3}$. При таких параметрах для времени нагрева электронов и пространственного масштаба $x_{T n}=\left(3 \sqrt{\pi / 2} v_{e n}(0) t_{T n}\right)^{1 / 2} c / \omega_{L e}$ получаем оценки $t_{T n} \sim 0.1 \mu \mathrm{s}, x_{T n} \sim 2 \mathrm{~cm}$.

\section{Список литературы}

[1] Wharton C.B., Horn P., Robertson S. // Phys. Rev. Lett. 1971. Vol. 27. N 8. P. 499-501.

[2] Nishida Y., Hirose A., Skarsgard H.M. // Phys. Rev. Lett. 1977. Vol. 38. N 12. P. 653-656.

[3] Hirose A., Kawabe T., Skarsgard H.M. // Phys. Rev. Lett. 1972. Vol. 29. N 21. P. 1432-1434.

[4] Bychenkov V.Yu., Frank P., Eimmel G. et al. // Phys. Lett. A. 1992. Vol. 169. N 1, 2. P. 77-81.

[5] Быченков В.Ю., Новиков В.Н. // Физика плазмы. 1994. т. 20. Вып. 5. С. 513-516.

[6] Овчинников К.Н., Урюпин С.А. // ЖТФ. 2009. Т. 79. Вып. 7. C. 59-62.

[7] Овчинников К.Н., Силин В.П., Урюпин С.А. // Физика плазмы. 2009. Т. 35. Вып. 12. С. 1118-1125.

[8] Овчинников К.Н., Силин В.П., Урюпин С.А. // ЖТФ. 1989. Т. 59. Вып. 9. С. 29-36.

[9] Овчинников К.Н., Урюпин С.А. // Физика плазмы. 2013. Т. 39. Вып. 9. С. 837-847.

[10] Виноградов Н.И., Извозчиков А.В., Шаховеи К.Г. // Препринт ФТИ им. А.Ф. Иоффе № 1177. 1987.

[11] Гинзбург В.Л., Гуревич А.В. // УФН. 1960. Т. LXX. Вып. 2. C. 201-246.

[12] Базелян Э.М., Райзер Ю.П. Физика молнии и молниезащиты. М.: Физматлит, 2001. 320 с. 